\title{
A DECISION SUPPORT SYSTEM FOR EVALUATING QUALITY SAFETY RISK CONTAMINATED BY WATER POLLUTION IN AQUACULTURE POND
}

Dong Tian ${ }^{1,2}$, Nan $\mathrm{Li}^{1,2}$, Honghui Huang ${ }^{3}$, Zetian $\mathrm{Fu}^{1}$, XiaoShuan Zhang ${ }^{1, *}$

${ }^{1}$ China Agricultural University, Beijing, P. R China, 100083

${ }^{2}$ Key Laboratory for Modern Precision Agriculture System Integration at China Agricultural University, Ministry of Education, P. R. China, 100083

${ }^{3}$ Key Laboratory for Fishery Ecology Environment, Ministry of Agriculture, P. R China, 510300

* Corresponding author, Address: China Agricultural University, P. R. China,100083, Tel: +86-10-62736717,Email:td_tiandong@cau.edu.cn

Abstract: Water pollution is becoming the major factor damaging the sustainable development of aquaculture and the quality security of aquatic product in China. This paper introduces a decision support system for evaluating and managing quality risk contaminated by water pollution. The architecture, main components and their functions, especially a series of risk evaluation methods and models are described. At present, the system is in pilot in the city of Beijing in China. The stage achievements in developing the system are summarized.

Keywords: Decision support system, quality safety risk, water pollution, Aquaculture, evolutionary prototyping

\section{INTRODUCTION}

Aquaculture integrated into the smallhold farming system has been shown to improve both productivity and cash flows with little or no external

Please use the following format when citing this chapter:

Tian, D., Li, N., Huang, H., Fu, Z. and Zhang, X., 2009, in IFIP International Federation for Information Processing, Volume 293, Computer and Computing Technologies in Agriculture II, Volume 1, eds. D. Li, Z. Chunjiang, (Boston: Springer), pp. 643-652. 
input(Brummett and Noble,1995), is the fastest growing food-producing sector in the world and provides for almost 50 percent of the world's food fish requirements. As ocean stocks of wild fish diminish and fishing quotas are more strictly enforced, so farm-reared fish will continue to supply greater proportion of the growing consumption of aquatic food. Especially China produces about 70 percent of the farmed fish in the world, harvested at thousands of giant factory-style farms and has been transformed into the fastest-growing, biggest producer and exporter of fishery in the world over the past two decades.

But that growth is threatened by the two most glaring environmental weaknesses in China: acute water shortages and water supplies contaminated by sewage, industrial waste and agricultural runoff that includes pesticides, fish contaminated with toxic chemicals like DDT are already creating health problems. The fish farms, in turn, are discharging wastewater that further pollutes the water supply. Sometime Farmers have coped with the toxic waters by mixing illegal veterinary drugs and pesticides into fish feed, which helps keep their stocks alive yet leaves poisonous and carcinogenic residues in seafood, posing health threats to consumers(DAVID BARBOZA,2007).

Environmental degradation, in other words, has become a food safety problem, and the long-term risks of consuming contaminated fishery product could lead to higher rates of cancer and liver disease and other afflictions(DAVID BARBOZA,2007). Therefore, water quality pollution management are popular and important topics of fishery water environmental research due to the food quality security and health impact caused by exposing to water pollutants existing in aquaculture and preventive as well as evasive action execution during the episodes of waterborne pollution.

Obviously, there is an increasing interest and pressing need in an environmentally friendly culture to reach the development and dissemination of a range of fish culture systems that are both environmentally and economically sustainable. This paper introduces the development experience on the decision support system for quality risk evaluation and management contaminated by water pollution(WatPol) in aquaculture. The following is organized as: section 2 describes the development process; then the last section draws the conclusions and further improvement.

\section{THE DEVELOPMENT PROCESS}

For assuring ultimate success for developing WatPol, the evolutionary prototype mode had adopted as the development process or methodology taking into account prototyping process provides users with more opportunities to improve their work, to verify that their needs are provided 
for, and that the terms used in the interface of the designed system are consistent with those in use in their work.

The following milestones outline the major tasks undertaken to develop the WatPol. A number of the tasks overlapped. The project was initiated in 2006 and the prototype was finished in 2007, which development process illustrates in Fig 1.

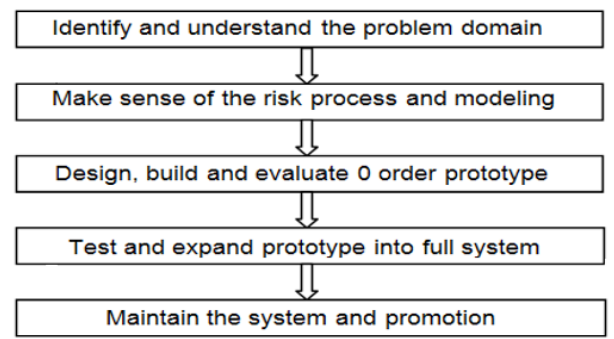

Figl. the development process based on the prototyping model for WatPol

\subsection{Identify and understand the problem domain}

The first step in the project is to identify and understand the problem domain. It was the most complex and time-consuming part of the entire process. It requires working with many organizations or experts in order to get a handle on the structure and the content of expert system and determine the information that allow the problem domain to be defined correctly. The quality at this process leads directly to and impacts the success of last steps.

The problem had been identified by multiple methods in Beijing, Tianjin and Shandong:

1) Laboratory experiments. Fish are totally dependent upon water to breathe, feed and grow, excrete wastes, maintain a salt balance, reproduce, and understanding the physical and chemical qualities of water is critical to successful aquaculture. To a great extent water determines the success or failure of an aquaculture operation(LI Nan, et al,2007). Laboratory experiment aims not only to identify and rank the factor influenced the water quality and fish quality risk, but also to acquire the daily fluctuation via monitoring of pond water quality.

2) Experts questionnaires and interviews. The above methods can identify the relationship between water quality and fish quality safety, but it can't provide a risk management process and give a threshold for risk evaluation. The expert questionnaires and interviews aimed to further rank and weight the water quality factors, acquire the criteria for fish quality safety and the management process and strategies to reduce the losses. In the project, 22 experts from the domains of water quality and fish quality safety management were involved in a multi-step process of knowledge acquisition 
by the method of Delphi. The response rate from experts was $65 \%$. Reasons for this was that they were not interested or they could not find enough time as completing it took nearly one hour and must carefully think. Table 1 shows basic thresholds aquired from questionnaires.

Table 1. the grade of water eutrophication assessment

\begin{tabular}{ll}
\hline Grade & Trophic status(EI) \\
\hline benchmark & {$[0-10]$} \\
I & Innutritive(10-13] \\
II & Nutritive(13-20] \\
III & Low-eutrophic(20-35] \\
IV & Medium-eutrophic(35-80] \\
V & High-eutrophic(80-95] \\
\hline
\end{tabular}

3) Literature review. A literatures review on water quality pollution, fish quality safety risk and modeling methods were conducted to provide a supplemental source of knowledge during system development to overcome the difficult caused by incomplete knowledge and data shortage. So that, the knowledge engineer could compare the different methods and develop the most appropriate and effective models to evaluate and forecast the water pollutant and fish quality safety risk.

\subsection{Make sense of the risk evaluation and modeling}

Based on the analysis on the information from step 1, the system development engineer and experts met several times to further understand the general practices more experts had accumulated the experience with the water quality and quality safety risk. The process is iterative and includes the following steps and models/methods.

Step 1 the model/method for identifying the primary water environmental pollutants factors in Aquaculture pond. PCA(Principal component analysis) method was adopted to identify the primary water pollutant factors and reduce multidimensional data sets to lower dimensions for analysis.

Step2 the model/method for analyzing the relationship between water environmental pollutants and fish quality safety risk. The linear regression was adopted to model the relationship between two variables by fitting a linear equation to observed data. The selected of water environmental pollutants was considered to be an explanatory variable, and the quality safety risk index was considered to be a dependent variable.

Step3 the model/method for predicting the trend of water environmental pollutants acclimated in Aquaculture pond. Support vector machines (SVMs), a novel type of learning machine based on statistical learning theory, was adopted as the basic algorithm due that SVM achieves an optimum network structure by striking a right balance between the empirical error and 
the VC-confidence interval. This balance eventually leads to better generalization performance than other neural network models(LI Nan, et al,2007).

Step4 the model/method for evaluating the probability the quality safety risks contaminated by water pollution. Monte Carlo methods were adopted as the basic methods. It is a class of computational algorithms that rely on repeated random sampling to compute their results. Because of their reliance on repeated computation and random or pseudo-random numbers, Monte Carlo methods are most suited to calculation by a computer. Monte Carlo methods tend to be used when it is infeasible or impossible to compute an exact result with a deterministic algorithm.

\subsection{Design, build and evaluate 0 order prototype}

\subsubsection{Design and develop the prototype}

A prototype system was built to test the functional specifications. Meetings with the system development engineer and experts were held until a consensus was reached on the objectives, constraints and specific tasks that the WatPol must perform to accomplish the design objectives.

$\mathrm{B} / \mathrm{S} / \mathrm{S}$ (Browser/Server/Server), which is able to better apply information system to network production, hardware platform and net compromise setting in different factories, was adopted as our system's fundamental architecture to provide the transparency among the data layer, business logic layer and the user interface layer(ZHANG Xiaoshuan, etc,2006).

The models/methods developed in step 2 to perform the tasks had been coded into modules. Each module was described in terms of its basic algorithm and the required inputs, outputs, and knowledge sources(Fig 2, Fig $3)$. Benchmarks were established for completing the different modules. The end result was a design document, which aided in the planning and programming of the WatPol.

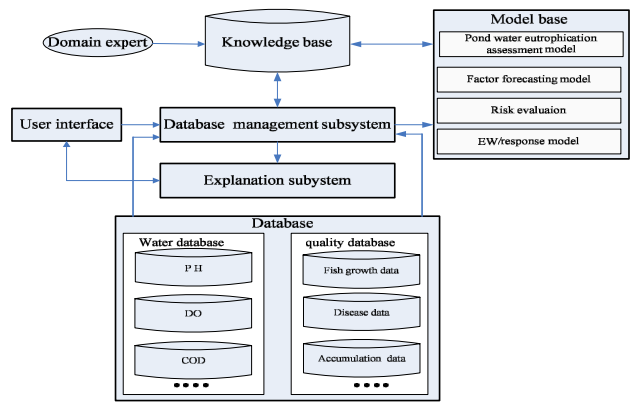

Fig 2. General framework of the software 


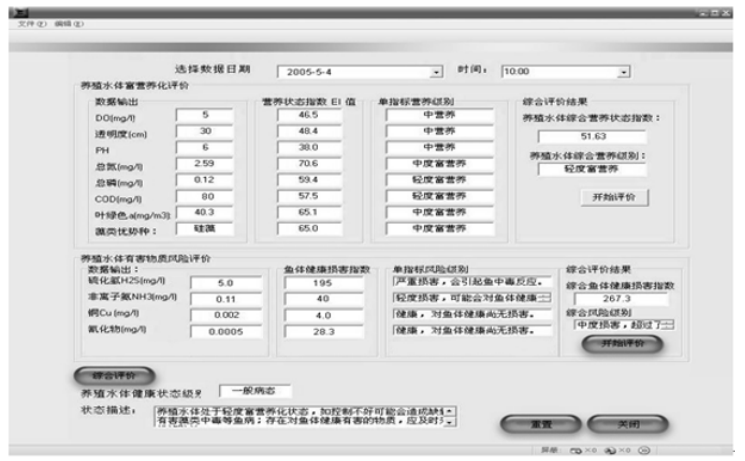

Fig3. the interface of water eutrophication assessment

\subsubsection{Evaluate 0 order prototype}

Prototype evaluation process is fundamental in order to ensure the quality of the system and be convenient to the prototype expand the full system. After finished the Version 1.0, the system were promoted in several ways given that web-based systems had shown some benefits to easily accessible by users, enhance traditional explanation and justification functions with hyperlinks to other relevant sites and involve users in validation process.

- One was to send the introduction of the WatPol by emails or/and mails to the potential user and guide them directly logon on the website;

- Second was some workshops and trainings were held unusual;

- Another was to collect of user feedback via online forms and chat with the users via internet.

\subsection{Test and expand prototype into full system}

Based upon the design document, the prototype was expanded into the complete system. The actual and experiment data had been collected for the system test. Some users' feedback had collected. The following focuses the test based on the experiment data.

\subsubsection{Experiment data collection}

For evaluating and testing the system, a series experiments had been executed to acquire the test data from April to October,2006 in Beijing.

The water was sampled both from the external water suppliers and pond internal water. Not only the physical and chemical elements, but also the species composition including predominate species and biomass of planktons were sampled and tested. 
The second experiment was responsible for collecting the daily data of the selected factors. It lasted from April to September in 2007. Water was sampled three times per day at 8:00 am, 14:00 pm and 20:00 pm respectively.

The last experiment was responsible for analyzing the fish quality safety. The pollutants factor found in the first experiment maybe accumulate in fish body and trigger oxidative stress in fish and affect their growth (J.E. Hinck, 2006). Nearly twenty fish per week were caught at random in each of the ponds. After capture, the livers, which was the main detoxification organ in fish (G. Deviller, et al., 2005), were dissected and weighed.

\subsubsection{Data analysis and modeling}

Step 1 identifying the primary water environmental pollutants factors

The result showed that the pond water was rich nutritive and the main pollutant of the pond water were non-ionic ammonia (NH3), sulfureted hydrogen $(\mathrm{H} 2 \mathrm{~S})$, copper $(\mathrm{Cu})$, cyanide $(\mathrm{HCN})$ and Coliform during the experiment period.

In addition, after qualitative and quantitative analysis of phytoplankton, the results turned out that ninety-eight kinds of algae were detected in the pond, in which, 53\% was Chlorophyta species, 34\% was Bacillariophyta species, $8.2 \%$ was Cyanophyta species, $3.3 \%$ was Cryptophyta species and $1.5 \%$ was pyrrophyta species.

Taking the COD as example, the concentration of CODcr in the pond water is affected by a series of factors, such as dissolved oxygen (DO), water temperature, water hardness (WH), nitrogen, phosphorus and so on. In this research, the principal factor analysis is used to select the most important factors as the input variables of the SVM network. The result of the principal factor analysis is shown in Table 2 .

It can be concluded from the correlation analysis in Table 2 that water temperature (T), DO, and water hardness ( $\mathrm{WH}$ ) have remarkable correlations with CODcr, which means that the variation of them will affect the concentration of CODcr in the pond water. It also can be seen from Table 2 that the principal component coefficients of DO, water temperature and CODcr are higher than other factors in the first component. Although the principal component coefficients of water hardness and total phosphorus are high in the second and the fourth component, the contribution percentage is not high enough. 
Table 2. Principal component analysis of pond water quality factors

\begin{tabular}{cccccccc}
\hline & \multicolumn{3}{c}{ Principal component coefficient } & & Eigenvalue & Cumulative \\
\cline { 2 - 5 } & PH & T & DO & SD & & & \\
\hline 1 & -0.16 & 1.14 & 1.26 & 0.07 & 0.30 & 3.16 & 0.40 \\
2 & 0.46 & -0.15 & -0.04 & 0.44 & 0.73 & 1.48 & 0.58 \\
3 & -0.22 & -0.49 & 0.17 & -0.15 & 0.54 & 1.10 & 0.72 \\
4 & 0.05 & 0.10 & -0.20 & 0.44 & -0.17 & 0.94 & 0.84 \\
\hline
\end{tabular}

Step 2 analyzing the relationship between water environmental pollutants and fish quality safety risk(See Table 3 ).

Table 3. the regression model

\begin{tabular}{llll}
\hline Step & $\mathrm{r}^{2}$ & Paremeter & The regression model \\
\hline 1 & 0.53 & $\mathrm{X} 1=\mathrm{C}_{\mathrm{w}}$ & $\mathrm{Y}=0.32+0.58 * \mathrm{X} 1$ \\
2 & 0.61 & $\mathrm{X} 2=\mathrm{PH}$ & $\mathrm{Y}=0.36+0.61 * \mathrm{X} 1-0.2 \mathrm{X} 2$ \\
3 & 0.69 & $\mathrm{X} 3=\mathrm{WH}$ & $\mathrm{Y}=0.46+0.53 \mathrm{X} 1-0.16 \mathrm{X} 2-0.00321 \mathrm{X} 3$ \\
4 & 0.74 & $\mathrm{X} 4=\mathrm{FW}$ & $\mathrm{Y}=0.5+0.47 \mathrm{X} 1-0.13 \mathrm{X} 2-0.00356 \mathrm{X} 2-0.045 \mathrm{X} 4$ \\
\hline
\end{tabular}

Where, $\mathrm{CW}$ is the $\mathrm{Cu}+$ density in water, $\mathrm{WH}$ is water hardness, $\mathrm{FW}$ is fish weight, $\mathrm{PFC}$ is forecasted $\mathrm{Cu}+$ density in fish issue 。

Step3 the model/method for predicting the trend of water environmental pollutants acclimated in Aquaculture pond.

Therefore, in this prediction experiments, DO, water temperature and CODcr are adopted as the input variables of the SVM network. In order to validate the feasibility of our hypothesis, five sets of input variables are considered and experimented, and the output is the level of CODcr in the next week. With comparing the Mean absolute error (MAE) and the Square root error, the best set of input variables is selected ultimately.

Fig 4 shows that the anova kernel has better generalization performance than other kernel functions on the testing process, which is in consistency with statistical learning theory.

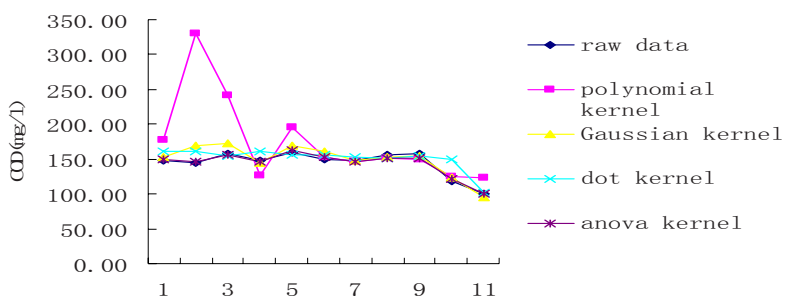

Fig 4. the SVM Kernel function selection

Step4 evaluating the probability of the quality safety risks contaminated by water pollution(Fig 5). It shows that uncertainties in the higher percentage point above grown exponentially. The risk of cumulative distribution function showed that the risk of contamination of aquatic products is less than $24 \%$ of the probability is 50 percent, 90 percent of the 
probability of contamination of the instructions for the higher risk of 95 percent. Risk in $25 \%$ of the growth index that pollutants in the fish concentration is a positive deviation lognormal distribution.

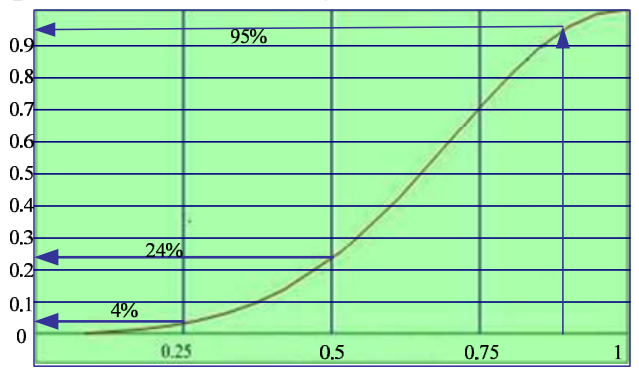

Fig 5 . the probability evaluation of the quality safety risks contaminated by water pollution

\subsubsection{The feedbacks from the experiment and test}

The experiment test proves the system as an aid and a valuable companion for improving the quality of decisions making of the farmers and the extension officers involved in the fish quality safety and water environment management at farm level. The followings discuss and conclude some benefits and shortcomings for applying the system:

The benefits are the system can help to reduce economical losses due to fish quality safety risk by its continuous monitoring and real-time measurement of water quality and pollution with the characteristics of flexibility to adapt to users' requirements and increasing process efficiency.

The shortcomings are 1) WatPol is just applicable to Beijing given that the selected factors and the corresponding criteria are confirmed based on the special area. 2) WatPol can't evaluate the quality safety risk contaminated by water pollution not the feed and vermin.

In addition, the system should collect automatically or semi automatically the cases of fish quality safety and water pollution as much as possible. Then the system can be more effective by integrating more artificial intelligence, such as case-based reasoning.

\subsection{Maintain the system and promotion}

A long-term support and maintenance plan was devised. The system was designed so additional information or modules could be easily added. The WatPol is marketed, through Beijing Kuntai software Co. Ltd, a private soft engineering company that is interested in expanding its business interests in aquaculture and software systems. The company is responsible for marketing and distributing the software, as well as, providing technical software 
support.

\section{CONCLUSION}

In this paper we develop multitasking software provides the ultimate in versatility for use in pond water pollution assessment, factor forecasting and fish quality safety risk assessment. The system can be used easily to explore the effect of different risk-reduction strategies.

Due that the factors are arbitrarily derived from case area, the system may not reflect reality all other of fish ponds. Whether the system is correct or not, the demonstration proved it was a powerful aid for teaching the principles of fish safety and can help train food safety managers to think in term of water pollution and fish safety risk.

The system is only seen as a prototype, not a definitive model. It needs be modified to suit the more broadly area and scenarios.

\section{ACKNOWLEDGEMENTS}

This program is supported by 863 Plan (No.2006AA10Z267), NSFC(30700481)and Open Fund from the Key Laboratory of Fishery Ecology Environment, Ministry of Agriculture, P. R China (No. 2006-9) and key laboratory of fishery water treatment, CAFS(No. FWT-200603).

\section{REFERENCES}

Brummett and Noble, 1995. Aquaculture for African smallholders. ICLARM technical report No. 46, pp. 69

DAVID BARBOZA, In China, Farming Fish in Toxic Waters, the new York times, December 15,2007

G. Deviller et al. Impact assessment of various rearing systems on fish health using multibiomarker response and metal accumulation. Ecotoxicology and Environmental Safety 6190 (2005) 89-97

J.E. Hinck et al. Environmental contaminants and biomarker responses in fish from the Columbia River and its tributaries: Spatial and temporal trends.Science of the Total Environment 366 (2006) 549-578.

LI Nan, et al. Using support vector machines to predict the variation of organic pollutants in pond water. Third International Conference on Natural Computation, ICNC 2007, 2007, p 697-701

ZHANG Xiaoshuan, etc. Developing a Web-based Early Warning System for Fish Disease based on Water Quality Management. 1st IEEE Conference on Industrial Electronics and Applications, 2006, p 4025733 